\title{
Wall-Modeling Large Eddy Simulation on rough wall for High Reynolds Number Flow
}

\author{
LIU $\mathrm{Yu}^{1, \mathrm{a}^{*}}$, TONG Mingbo ${ }^{1, \mathrm{~b}}$, CHEN Binqi ${ }^{1, \mathrm{c}}$ and WANG Fangli, ${ }^{1, d}$
}

${ }^{1}$ College of Aerospace Engineering, Nanjing University of Aeronautics and Astronautics, Nanjing 210016, P. R. China

aliuyuyyyy@126.com, btongw@nuaa.edu.cn, chenbinqi7@163.com, 'wangfangli2010@163.com

Keywords: Wall-Model, LES, boundary layer, rough wall, wall shear stress.

Abstract. In this work a wall-modeled LES is developed to solve the basic flow character over rough wall on high Reynolds number without resolving the details of the roughness elements. The averaged roughness shear stress can be combined with the smooth wall shear stress in the inner layer wall-model. The instantaneous shear stress caused by the roughness elements are calculated by the pressure projection and the elevation fields. The total shear stress is given back to the outer layer LES mesh as a new boundary condition. The comparison between simulation and the experiment reveals that the wall-modeled LES approach in this research is capable to predict the flow in rough wall boundary layer without resolving the detailed roughness element geometries.

\section{Introduction}

It is well known that the computational cost of large eddy simulation (LES) on turbulent boundary layer at high Reynolds numbers is enormous. The grid resolution will be extremely high when using LES to resolve the inner layer motions. Therefore, the inner layer should be modeled while the outer layer could be directly resolved by LES in high Reynolds number wall-bounded flows.

In the wall-modeling LES approach [1, 2], the wall-model grid which covers the inner boundary layer is generated by extruding the wall surface mesh along the normal direction of the wall surface. The total wall shear stress $\tau_{\mathrm{w}}$ on rough wall can be divided into two parts, the smooth wall shear stress $\tau_{\mathrm{sw}}$, the roughness shear stress $\tau_{\mathrm{rw}}$. If the skin shear stress which will be given back to LES in the wall model can combine both the smooth wall shear stress and shear stress caused by roughness element, the roughness element will not be resolved by either the LES grid or wall-modeling grid.

The unsteady shear stress which is considered as instantaneous shear stress $\tau_{\text {rw }}^{\prime}$ obtained by the unsteady drag have great influence on the noise generation of rough wall. In light of this observation, the roughness shear stress $\tau_{\mathrm{rw}}$ can be divided into the averaged effect of roughness element $\bar{\tau}_{\mathrm{rw}}$ and the instantaneous roughness shear stress $\tau_{\mathrm{rw}}^{\prime}$ in the present work. The instantaneous shear stresses on rough walls are obtained by projecting the unsteady pressure at the edge of the inner layer onto the surface geometry of the roughness elements. This approach is schematically illustrated in Fig. 1.

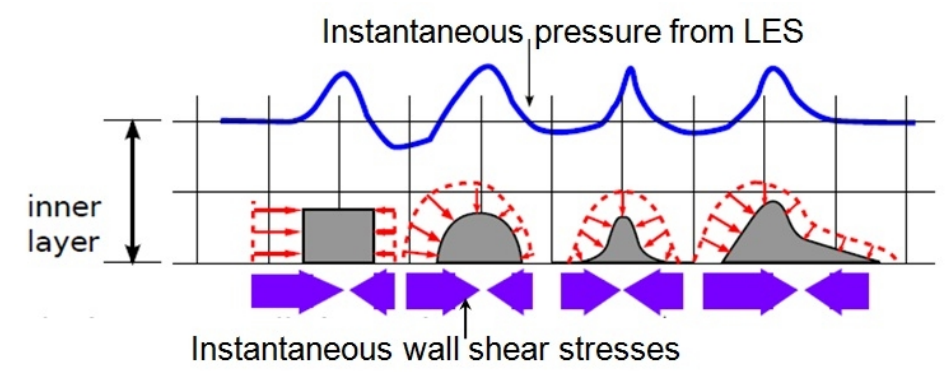

Figure 1 Rough wall model proposed in the present work

This novel rough wall model can solve rough wall boundary layer by three sets of mesh, the outer layer LES mesh, inner layer wall-modeling mesh and a surface mesh on the wall. The instantaneous wall shear stress caused by the roughness element can be calculated by the surface mesh on the wall instead of modeling the detailed geometry of roughness element. The wall-modeling mesh is used to estimate the smooth wall shear stress and return the total shear stress back to the outer layer LES mesh. 
Due to the wall-modeling mesh, the LES mesh could be coarse with the size of $\Delta \mathrm{x}^{+}, \Delta \mathrm{y}^{+}$and $\Delta \mathrm{z}^{+}$in stream-wise, wall-normal, and span-wise directions respectively, as large as 200 to 1200.

\section{Wall-modeling LES Approach on rough wall}

The inner layer wall model is a filtered equation [3]. Assuming equilibrium, the unresolved inner layer is modeled by solving:

$$
\frac{d}{d \eta}\left(\left(v+v_{t, w m}\right) \frac{d u_{\|}}{d \eta}\right)=0
$$

where $\eta$ is the wall-normal direction, which should usually be aligned with y direction for regular geometries; $\mathrm{u}_{\|}$is wall parallel velocity magnitude; $v$ is the kinematic viscosity. The kinematic eddy viscosity $v_{t, w m}$ is obtained from the mixing-length model [4]:

$$
v_{t, w m}=\kappa \eta \sqrt{\tau_{s w} / \rho}\left[1-\exp \left(-\frac{\eta^{+}}{\mathrm{A}^{+}}\right)\right]^{2}
$$

where $\mathrm{A}^{+}=17 ; \kappa=0.41$ is the von Karman constant. The wall parallel velocities from the instantaneous LES solution at $\eta=h_{w m}$ are interpolated to the upper boundary of the wall-model mesh. The wall-shear stress $\tau_{\mathrm{sw}}$ is determined from the wall gradient of the inner solution [3]:

$$
\tau_{s w}=\left.\rho v \frac{d_{\|}}{d \eta}\right|_{\eta=0}
$$

Note that the inner layer equations can only calculate the smooth wall shear stress $\tau_{\text {sw }}$. Because the roughness elements are not resolved by the LES mesh, the averaged roughness shear stress $\overline{\tau_{\mathrm{rw}}}$ is involved through the relation with the smooth wall shear stress $\tau_{\mathrm{sw}}$. A proportion $\alpha$ is proposed as:

$$
\left.\alpha=\overline{\tau_{\mathrm{rw}}} /_{\left(\tau_{\mathrm{sw}}\right.}+\overline{\tau_{\mathrm{rw}}}\right)
$$

The parameter $\alpha$ can be determined from the experiments of previous research such as Blake [5] and Varano [6]. After the wall-model getting the smooth wall shear stress $\tau_{\mathrm{sw}}$, the total averaged wall shear stress can be obtained. The wall-model mesh required by solving the wall-model equation is created using a simple extrusion of the wall surface by a thickness of $h_{w m}=0.1 \delta$. According to the recommendation of Kawai and Larsson [7], five and a half LES cells are placed within the wall-model layer. The procedure to couple the LES with the wall-model is showed in Fig. 2.

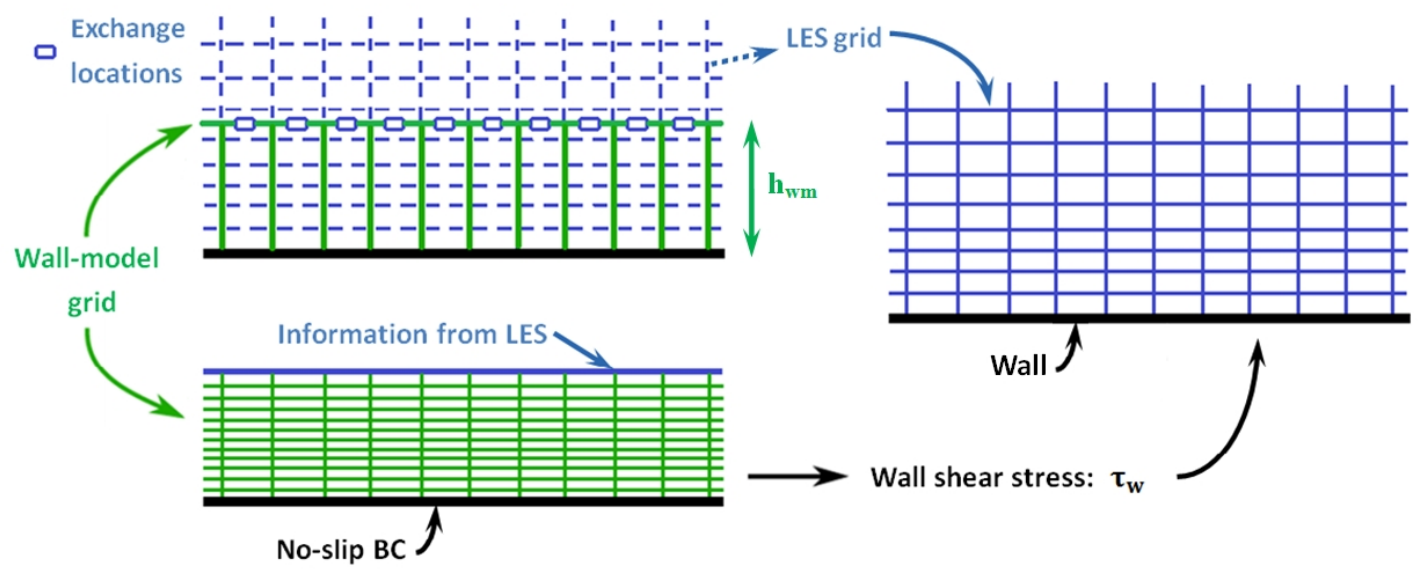

Figure 2 Couple of wall-model and LES

A third mesh besides the LES and wall-model mesh is set up to cover the roughness element, which only have one layer mesh in wall normal direction and dense grid distribution on stream-wise 
and span-wise directions. On the roughness mesh, the roughness geometry is adequately represented via an elevation field. Each roughness element can be covered in $\mathrm{x}$ and $\mathrm{z}$ directions by 3 grid points or more. As a result, the CFD mesh does not resolve the detailed roughness element geometries at all.

As seen in Fig. 1, assume the pressure distribution around the roughness element is $\mathrm{p}(\mathrm{x}, \mathrm{z})$, and the roughness elevation field is $h(x, z)$. A finite element $d A$ is taken from the surface of the roughness element, then the normal direction of the area is:

$$
\overrightarrow{\mathrm{n}}=\left(-\frac{\partial \mathrm{h}}{\partial \mathrm{x}}, 1,-\frac{\partial \mathrm{h}}{\partial \mathrm{z}}\right) / \sqrt{\left(\frac{\partial \mathrm{h}}{\partial \mathrm{x}}\right)^{2}+1+\left(\frac{\partial \mathrm{h}}{\partial \mathrm{z}}\right)^{2}} \text {, with }|\mathrm{dA}|=\sqrt{\left(\frac{\partial \mathrm{h}}{\partial \mathrm{x}}\right)^{2}+1+\left(\frac{\partial \mathrm{h}}{\partial \mathrm{z}}\right)^{2}}
$$

The force on infinite decimal element $\mathrm{dA}$ is

$$
\mathrm{d} \overrightarrow{\mathrm{F}}=-\mathrm{p}(\mathrm{x}, \mathrm{z}) \cdot \mathrm{dA}=-\mathrm{p}(\mathrm{x}, \mathrm{z}) \cdot\left[-\frac{\partial \mathrm{h}}{\partial \mathrm{x}}, 1,-\frac{\partial \mathrm{h}}{\partial \mathrm{z}}\right]=\mathrm{p}(\mathrm{x}, \mathrm{z}) \cdot\left[\frac{\partial \mathrm{h}}{\partial \mathrm{x}},-1, \frac{\partial \mathrm{h}}{\partial \mathrm{z}}\right]
$$

The projection in $\mathrm{x}$ and $\mathrm{z}$ directions are

$$
\tau_{\mathrm{x}}=\mathrm{dF}_{\mathrm{x}}=\mathrm{p}(\mathrm{x}, \mathrm{z}) \cdot \frac{\partial \mathrm{h}}{\partial \mathrm{x}}, \tau_{\mathrm{z}}=\mathrm{dF}_{\mathrm{z}}=\mathrm{p}(\mathrm{x}, \mathrm{z}) \cdot \frac{\partial \mathrm{h}}{\partial \mathrm{z}}
$$

which is the instantaneous roughness shear stress $\tau_{\mathrm{rw}}^{\prime}$ to be obtained. The total wall shear stress will then be got and given back to the LES mesh as a new boundary condition.

\section{Numerical Test for Rough wall model}

The experimental measurement of Varano [6] on rough wall plate in free-stream flow of $27 \mathrm{~m} / \mathrm{s}$ will be tested to evaluate the effective of the proposed method. The roughness elements are all made of 2 $\mathrm{mm}$ diameter hemispheres in a straight spacing in $11 \mathrm{~mm}$ as seen in Fig. 3. The roughness ratio $\alpha=0.135$ is determined from the experimental data.

The numerical test for the LES grid is $220 \mathrm{~mm}, \delta=60 \mathrm{~mm}$ and $110 \mathrm{~mm}$ in the stream-wise (x), wall-normal $(\mathrm{y})$ and span-wise $(\mathrm{z})$ directions, where $\delta$ is the boundary layer thickness in the experiment of Varano [6]. The Reynolds number based on $\mathrm{U}_{a n}$ and $\delta$ is $1 \times 10^{5}$. In viscous wall unit, the grids have $\Delta \mathrm{x}^{*}=\Delta \mathrm{z}^{*} \approx 180$. but varying $\Delta \mathrm{y}$ with the first layer from the wall $\Delta \mathrm{y}_{1}^{*} \approx 80$. Hence, the grid spacing is coarser than in traditional wall-resolved LES. The wall-modeling mesh which is used to calculate the smooth wall shear stress has the same grid as LES in $\mathrm{x}$ and $\mathrm{z}$ directions, but $\mathrm{y}=\mathrm{h}_{\mathrm{wm}} \approx 0.1 \delta$ and 100 points are distributed to resolve the velocity parallel to the wall and the wall shear stress. The roughness mesh only has one layer in y direction but 4 times of points in $\mathrm{x}$ and $\mathrm{z}$ directions to cover the roughness elements as shown in Fig. 3.
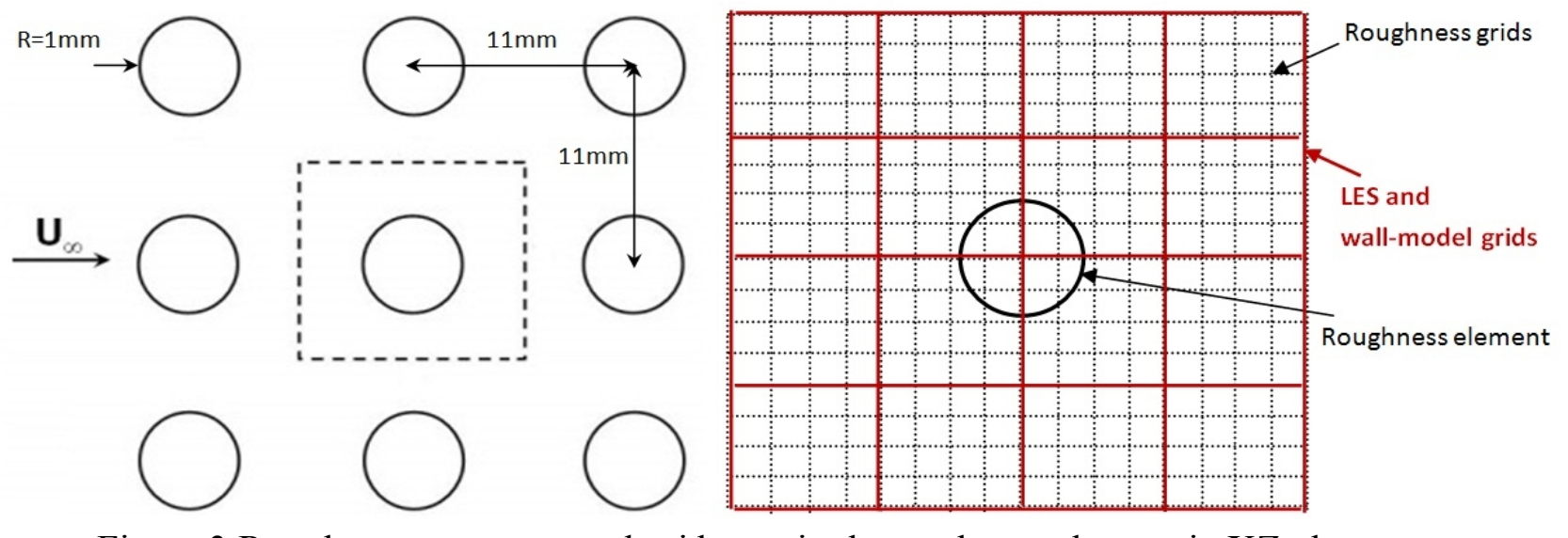

Figure 3 Roughness geometry and grids on single roughness element in XZ plane 
It should be noted that the numerical test is carried out on a half channel, the slip wall boundary condition is used on the upper boundary and cyclic flow conditions are chosen for the stream-wise and span-wise boundaries. The time step is $5 \mathrm{e}-5 \mathrm{~s}$ which makes the Courant number close to 0.5. The time unit is $0.008 \mathrm{~s}$ with the free-stream velocity $27 \mathrm{~m} / \mathrm{s}$. A preliminary computation is performed during 1s (125 time units) on the pure LES grid to get a reasonable mean flow around the wall. Wall-modeling LES computations are then integrated during 250 time units to wash out the initial transients, and the averages are collected over another 125 time units. The computation costs about 400 core-hours.

\section{Results and discussion}

The computed mean velocity and the normalized profile are shown in Fig. 4 and Fig. 5. In order to demonstrate the flow character of rough wall, the smooth wall results in the same flow condition are also shown with dotted lines. The wall-modeled LES approach has high accuracy on smooth wall flow prediction. Due to the effect of roughness elements, the mean velocity profile is produced a shift, which is caused by the drag on roughness element. This effect is also observed in the wall-modeled LES predictions. Although the roughness elements are not resolved through the wall-modeling or LES mesh, by artificially adding the averaged roughness shear stress into the total shear stress and giving it back to LES mesh, the out layer LES are capable to reflect the rough wall flow character. In light of this, the deviation in the LES region is smaller than in the wall-modeling region, as the inner layer does not have real roughness elements at all.

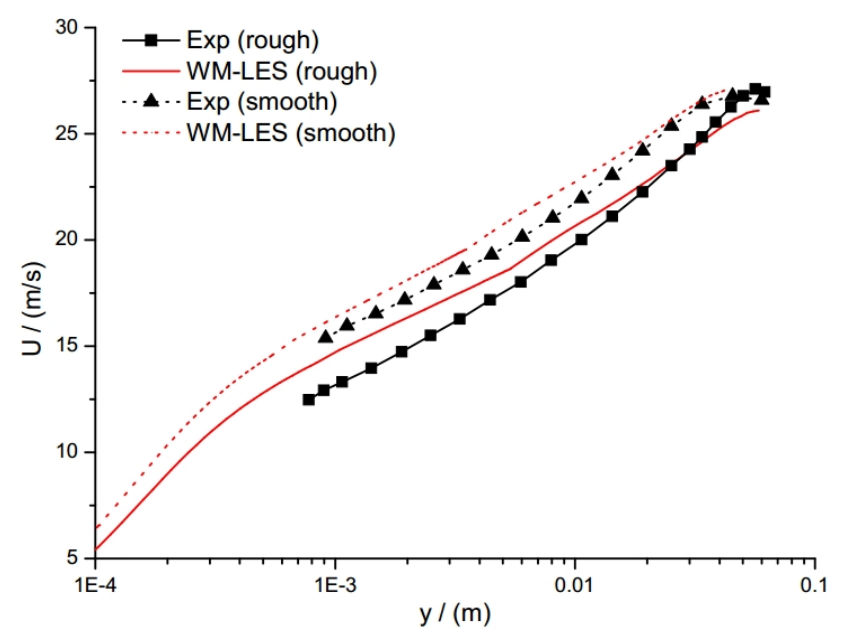

Figure 4 Mean velocity profile

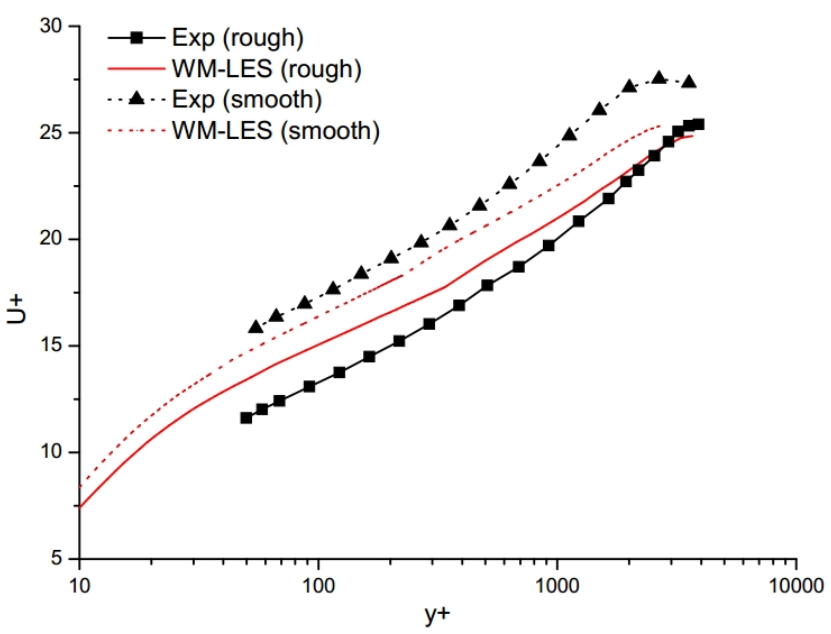

Figure 5 Normalized mean velocity profile

The shift also appears in the normalized mean velocity profile in Fig. 5, which is determined by both the mean velocity profile and the difference of friction velocity ${ }^{u_{\tau}}$. As discussed previously, the LES region agrees with the experiment data better than the wall-modeling region for the rough wall case, which means that the basic idea of wall-modeling LES approach on rough wall is promising. It should be noted that the friction velocity used in the experiment is obtained by the fitting methods which are highly dependent on the assumed value such as von Karman constant $\kappa$. Besides, the smooth wall von Karman constant is not appropriate for the rough walls. Therefore, the uncertainty associated with friction could be an important reason for deviation.

The Reynolds stresses are the reflection of turbulence fluctuation. As the most representative components, the stream-wise normal stress u'u' and Reynolds shear stress u'v' are shown in Fig. 6 and Fig. 7. According to the experiments, all the Reynolds stresses are normalized by inner scales, ${ }^{u_{r}}$, and $v / u_{\tau}$. As explained in the last section, only the outer layer LES results are compared with the experiments. The simulation results at lower $\mathrm{y}^{+}$region does not agree well with the experiments, however, most LES region shows the same trend with acceptable deviation. 


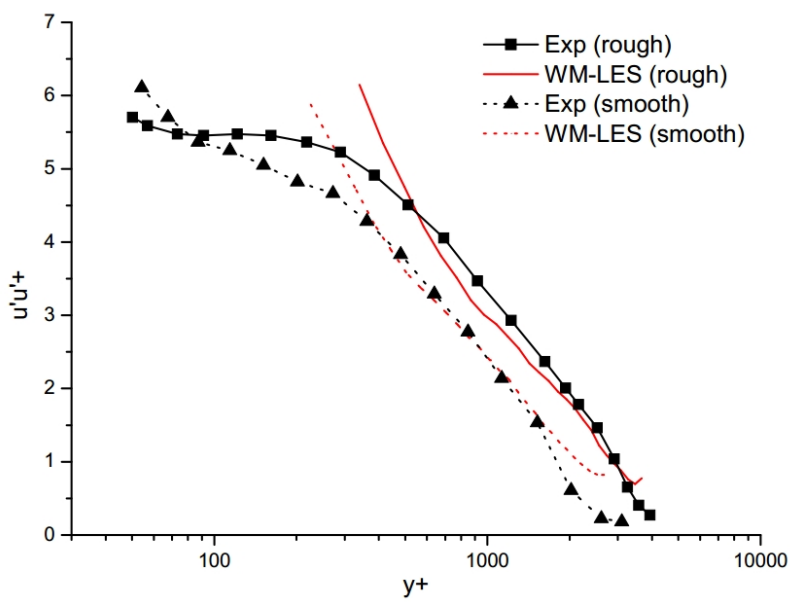

Figure 6 Normalized stream-wise normal stress

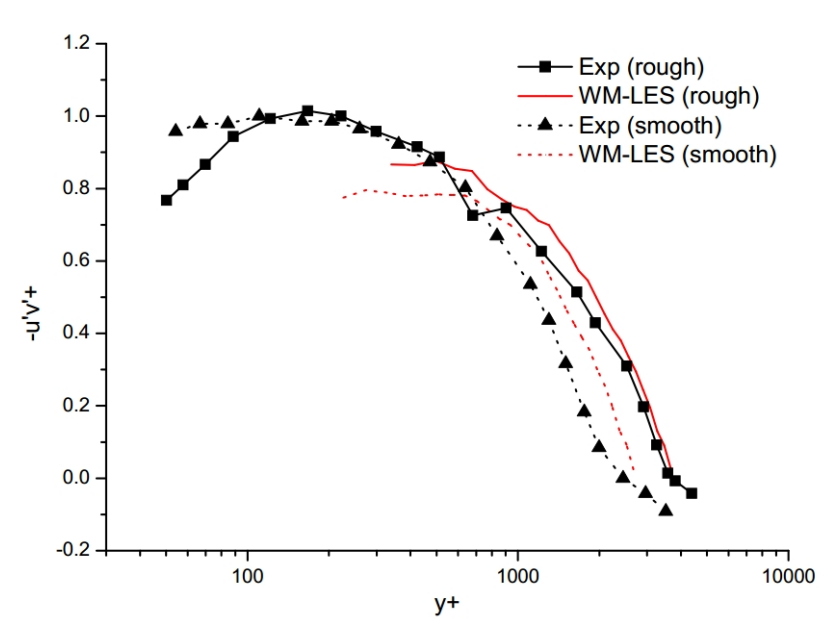

Figure 7 Normalized Reynolds shear stress

\section{Summary}

A novel boundary layer simulation is proposed, which is based on the wall-modeled LES approach. For the rough wall flow, the overall wall shear stress $\tau_{w}$ can be broken into the averaged smooth wall shear stress $\tau_{s w}$, the averaged roughness shear stress $\bar{\tau}_{r w}$ and the instantaneous roughness shear stress $\tau_{r w}^{r}$. After getting the total rough wall shear stresses, the wall-model will give it back to the LES mesh in the outer boundary layer as an updated boundary condition. The simulation on rough wall agrees with the experiment well and the wall-modeled LES approach in this research can predict the flow in rough wall boundary layer without resolving the detailed roughness element geometries.

\section{Acknowledgment}

This paper is a part of a project funded by the Priority Academic Program Development of Jiangsu Higher Education Institutions (PAPD).

This work is also supported by Funding of Jiangsu Innovation Program for Graduate Education KYLX_0296, and the Fundamental Research Funds for the Central Universities.

\section{References}

[1] U. Schumann, Subgrid scale model for finite difference simulations of turbulent flows in plane channels and annuli, J. Comput. Phys. 18, 376 (1975).

[2] E. Balaras, C. Benocci, U. Piomelli, Two-layer approximate boundary conditions for large-eddy simulations, AIAA J. 34, 1111 (1996).

[3] W. Cabot, P. Moin, Approximate wall boundary conditions in the large-eddy simulation of high Reynolds number flow, Flow, Turbulence and Combustion. 63, 269 (1999).

[4] W. Cabot, Large-eddy simulations with wall models, Annual Research Briefs: Center for Turbulence Research, Stanford, CA (1995) 41-50.

[5] Blake W, Turbulent boundary layer wall-pressure fluctuations on smooth and rough walls, J. Fluid Mech. 44 (1970) 637-660.

[6] Nathaniel D. Varano, Fluid dynamics and surface pressure fluctuations of turbulent boundary layers over sparse roughness, Ph. D Dissertation in Virginia Tech, Blacksburg (2010)

[7] Soshi Kawai, Johan Larsson, Wall-modeling in large eddy simulation: length scales, grid resolution and accuracy, Physics of Fluids, 24, 1 (2012) 015105+ 